\title{
Capsule commentary on Kurtzman et al., Social incentives and gamification to promote weight loss: the LOSE IT randomized, controlled trial
}

\author{
Samuel G. Smith, BSC, MSC, PhD \\ Leeds Institute of Health Sciences, University of Leeds, Leeds, UK.
}

J Gen Intern Med 33(10): 1782

DOI: $10.1007 / \mathrm{s} 11606-018-4603-7$

(c) The Author(s) 2018

$\mathrm{K}$ urtzman et al. report a randomized controlled trial testing the effectiveness of gamification interventions on weight loss among 196 adults with obesity. ${ }^{1}$ Participants formed teams with a family member or friend prior to randomization, and were given a goal of 10,000 daily steps and a weight loss goal of 6-8\% of baseline weight. Intervention participants also received a multi-component intervention involving weekly targets and entry into a team game. This involved the following: (1) a step count pledge, (2) weekly points linked with the pair's weigh-in adherence, and (3) a scoring system that promoted or demoted the pair to four levels based on weight loss. One intervention group also had their weight and step data shared regularly with their primary care physician. Financial incentives were provided to participants for completing weigh-ins.

There were within group differences in weight loss at 24 weeks among the intervention groups. However, in keeping with previous trials, ${ }^{2}$ weight loss also occurred within the control group. There were no significant differences in weight loss between each of the intervention arms and control at 12, 24 , or 36 weeks. Exploratory analysis indicated teammates living together achieved more weight loss than those not living together, particularly among the intervention conditions. This finding supports observational evidence, ${ }^{3}$ and shows promise for the design of future behavioral interventions. Replication in a trial fully-powered for this analysis is warranted.

The intervention was designed by incorporating multiple evidence-based components (e.g., gamification, incentives, targets, etc.), within a single package in the hope of maximizing behavior change. This classical approach is laudable for having the ultimate end-goal in sight: improved patient outcomes. However, when such trials yield null findings we are left with unanswered questions. Did any of the intervention components have an effect on the outcome? Were there antagonistic interactions of the intervention components on the outcome? The multiphase optimization strategy (MOST), ${ }^{4}$ suggests such questions should be addressed earlier using an optimization trial, such as a factorial experiment or sequential multiple assignment randomized trial (SMART). When designing behavioral interventions, consideration should be given to using MOST as a guiding framework.

Corresponding Author: Samuel G. Smith, BSc, MSc, PhD; Leeds Institute of Health Sciences University of Leeds, Leeds, UK (e-mail: s. smith1@leeds.ac.uk).

\section{Compliance with ethical standards:}

Conflicts of interest: The authors declare that they do not have a conflict of interest.

Open Access This article is distributed under the terms of the Creative Commons Attribution 4.0 International License (http:// creativecommons.org/licenses/by/4.0/), which permits unrestricted use, distribution, and reproduction in any medium, provided you give appropriate credit to the original author(s) and the source, provide a link to the Creative Commons license, and indicate if changes were made.

\section{REFERENCES}

1. Kurtzman GW, Day SC, Small DS, Lynch M, Zhu J, Wang W, Rareshide CAL, Patel MS. Social incentives and gamification to promote weight loss: the LOSE IT randomized, controlled trial. J Gen Intern Med. SPI 4552

2. Johns DJ, Hartmann-Boyce J, Jebb SA, Aveyard P, and on behalf of the Behavioural Weight Management Review Group. Weight change among people randomized to minimal intervention control groups in weight loss trials. Obesity. 2016, 24(4): 772-780.

3. Jackson SE, Steptoe A, Wardle J. The influence of partner's behavior on health behavior change: the English longitudinal study of ageing. JAMA Intern Med 2015: 175(3): 385-392.

4. Collins LM. Optimization of behavioral, biobehavioral and biomedical interventions: the multiphase optimization strategy (MOST). 2018. New York, NY, Springer. 